\title{
Existence of pseudo-equilibria in a financial economy
}

\author{
Philippe Bich and Bernard Cornet
}

Dedicated to Steve Smale on the occasion of his eightieth birthday

\begin{abstract}
This paper proves the existence of a pseudo-equilibrium in a financial economy with incomplete markets in which the agents may have nonordered preferences. We will use a fixed-point-like theorem of [4] that generalizes the results by Hirsch, Magill, Mas-Colell [18] and Husseini, Lasry, Magill [19] to encompass the framework considered by Gale and Mas-Colell $([14],[15])$.
\end{abstract}

Mathematics Subject Classification (2000). Primary 47H10, 91B50.

Keywords. Pseudo-equilibrium, incomplete markets, nonordered preferences, fixed-point-like theorems, Grassmann manifold.

\section{Introduction}

Since Arrow-Debreu [3], fixed-point theory has been a standard tool in general equilibrium theory to prove the existence of a Walrasian equilibrium. The first existence result has been derived from fixed-point theorems à la Brouwer-Kakutani in the case where agents have preferences represented by a complete preorder. The book Theory of value of Gérard Debreu [11] summarizes the work in the 50s, by Kenneth Arrow, Gérard Debreu, John Nash, John Von Neumann et Oskar Morgenstern, that essentially used arguments from general topology, convex analysis, together with the fixed-point theorems of Brouwer [6] and Kakutani [20]. The work by Gale and Mas-Colell ([14], [15]) then extended Kakutani's result to treat the existence problem when the agents have nonordered preferences. Another approach to the existence problem, which relies on degree theory or Poincaré-Hopf's theorem was initiated in the 70s by Steve Smale in a sequence of papers on "Global 
analysis and economics" (see [25],[26],[27],[28],[29]). This approach had a tremendous influence in the literature including the present paper and is presented in the books by Balasko [5], Dierker [10] and Mas-Colell [22]).

General Equilibrium Theory with Incomplete markets aims to study the interactions of the financial part of the economy and its commodity part in a world where time and uncertainty play a fundamental role (see, for example, Radner [24], Magill-Quinzii [21], or Geanakoplos [16] for a survey on GEI model). The mathematical tools used to prove the existence still rely on fixed-point-like theorems but of a different kind since some variables may belong to a Grassmann manifold $G^{k}(V)$, that is, the set of vector spaces of fixed dimension $k$ in a given Euclidean space $V$. The main difficulty to deal with this extended framework comes from the fact that, in general, the set $G^{k}(V)$ does not satisfy properties of convexity or acyclicity that would allow to use standard fixed-point theorems of Brouwer, Kakutani or Eilenberg-Montgomery [13] to deduce the existence of equilibria as in the standard case.

The aim of this paper is to prove the existence of a pseudo-equilibrium in a financial economy with incomplete markets in which the agents may have nonordered preferences and thus extend the existence result by Duffie-Shafer [12]. We will use a fixed-point-like theorem of [4] that generalizes the results by Hirsch, Magill, Mas-Colell [18] and Husseiny, Lasry, Magill [19] to encompass the framework considered by Gale and Mas-Colell ([14],[15]). The article is organized as follows. In Section 2, we present the general equilibrium model with incomplete markets and recall the definitions of an equilibrium and of a pseudo-equilibrium. We also state the main existence result (Theorem 1 ) for the existence of a pseudoequilibrium. The proof of the main existence result is given in Section 3 .

\section{The model and the main result}

\subsection{The model}

We first give the formal definition of the model. There is a finite set $I$ of consumers (by abuse of notation, $I$ will also denote the cardinal of the set $I$ ) and we consider the simplest case of intertemporal model with two dates, $t=0$ (today) and $t=1$ (tomorrow) (see [21], [1], [8] for a generalization to several periods). There is no uncertainty at date 0 , and the uncertainty at date $t=1$ is represented by finitely many states of nature $S(s=1, \ldots, S)$; only one state happens at $t=1$ and it is only known at date $t=1$. For convenience, the unique state of nature at $t=0$ will be denoted $s=0$. There is a positive number $\ell$ of divisible goods available at each date and each state $s=0, \ldots, S$; hence, the number of commodities available either at $t=0$ (with certainty) or at $t=1$ (contingent on each possible state of nature) is $L=\ell(1+S)$. An economy $\mathcal{E}$ can be summarized by a list $\mathcal{E}=\left(\left(X_{i}, P_{i}, e_{i}\right)_{i \in I}, V\right)$ where for every $i \in I$ : 
- $X_{i} \subset \mathbb{R}^{L}$ is the consumption set of the $i$-th consumer, that is the set of possible consumptions of this consumer.

- For all $x \in X:=\Pi_{i \in I} X_{i}, P_{i}(x) \subset X_{i}$ is the set of consumptions which are strictly preferred to $x_{i}$ by the $i$-th consumer, given the consumptions $\left(x_{j}\right)_{j \neq i}$ of the other consumers. ${ }^{1}$ Thus, $P_{i}$ is a correspondence from $X$ to $X_{i}$.

- $e_{i} \in \mathbb{R}^{L}$ is the initial endowment vector of the $i$-th consumer.

- At time $t=0$, there exist financial markets for a positive number $J$ of assets, $J \leq S$. An asset $j(j=1, \ldots, J)$ can be bought at time $t=0$ and delivers at time $t=1$ a financial payoff $V_{s}^{j}(p)$ (in unit of account) if state $s$ prevails, given the commodity price vector $p \in \mathbb{R}^{L}$. We denote by $V(p)=\left(V_{s}^{j}(p)\right)$ the $S \times J$ payoff matrix across states $s=1, \ldots, S$.

Given the asset price vector $q=\left(q_{1}, \ldots, q_{J}\right) \in \mathbb{R}^{J}$ specifying the price $q_{j}$ of the $j$-th asset for $j=1, \ldots, J$, we denote by $W(p, q)$ the $(1+S) \times J$ total payoff matrix across states $s=0, \ldots, S$, that is

$$
W(p, q)=\left(\begin{array}{c}
-q \\
V(p)
\end{array}\right) .
$$

The vector $z=\left(z_{1}, \ldots, z_{J}\right) \in \mathbb{R}^{J}$ denotes the portfolio of a consumer and specifies the quantity $\left|z_{j}\right|$ of each asset $j$ in the portfolio, with the usual convention that if $z_{j}>0$ then $\left|z_{j}\right|$ represents the quantity of asset $j$ bought at date 0 and if $z_{j}<0$ then $\left|z_{j}\right|$ represents the quantity of asset $j$ sold at date 0 . Then $W(p, q) z$ is a $(1+S)$-column vector (or by extension a vector in $\mathbb{R}^{1+S}$ ) which describes the financial stream of the portfolio $z$ across the $1+S$ states of nature.

\subsection{Equilibria and pseudo-equilibria}

We now formally define the notions of equilibrium and of pseudo-equilibrium. ${ }^{2}$

Definition 2.1. An equilibrium of the economy $\mathcal{E}$ is a list $(\bar{x}, \bar{z}, \bar{p}, \bar{q})$ in $\mathbb{R}^{L I} \times \mathbb{R}^{J I} \times$ $\mathbb{R}^{L} \times \mathbb{R}^{J}$ such that, if we let $\bar{x}=\left(\bar{x}_{i}\right)_{i \in I}$ and $\bar{z}=\left(\bar{z}_{i}\right)_{i \in I}$, one has:

(i) for every $i \in I,\left(\bar{x}_{i}, \bar{z}_{i}\right) \in B_{i}(\bar{p}, \bar{q})$ and $\left(P_{i}(\bar{x}) \times \mathbb{R}^{J}\right) \cap B_{i}(\bar{p}, \bar{q})=\emptyset$, where $B_{i}(\bar{p}, \bar{q})=\left\{\left(x_{i}, z_{i}\right) \in X_{i} \times \mathbb{R}^{J}: \bar{p} \square\left(x_{i}-e_{i}\right) \leq W(\bar{p}, \bar{q}) z_{i}\right\}$;

(ii) $\sum_{i \in I} \bar{x}_{i}=\sum_{i \in I} e_{i}$;

\footnotetext{
${ }^{1}$ This general way to describe the tastes of the consumers encompasses the case where the consumer $i$ has a preference relation $\preceq_{i}$ which is a complete preorder on $X_{i}$. In this case, for all $x \in \Pi_{i \in I} X_{i}, P_{i}(x)=\left\{x_{i}^{\prime} \in X_{i}: x_{i} \prec_{i} x_{i}^{\prime}\right\}$ where the strict preference relation $\prec_{i}$ is defined by $x_{i} \prec_{i} x_{i}^{\prime}$ if $\left[x_{i} \preceq_{i} x_{i}^{\prime}\right.$ and not $\left.x_{i}^{\prime} \preceq_{i} x_{i}\right]$.

${ }^{2}$ In this paper, we will often denote a vector $x \in \mathbb{R}^{L}$ by $x=(x(0), x(1), \ldots, x(S))$ where $x(s) \in$ $\mathbb{R}^{\ell}$ for $s=0,1, \ldots, S$. If $p=(p(0), \ldots, p(S)) \in \mathbb{R}^{L}\left[\right.$ resp. $\left.\lambda=(\lambda(0), \ldots, \lambda(S)) \in \mathbb{R}^{1+S}\right]$ and $x=(x(0), \ldots, x(S)) \in \mathbb{R}^{L}$, we denote $p \square x[$ resp. $\lambda \square p]$ the vector in $\mathbb{R}^{1+S}\left[\mathbb{R}^{L}\right]$ defined by $p \square x=(p(0) \cdot x(0), p(1) \cdot x(1), \ldots, p(S) \cdot x(S))[$ resp. $\lambda \square p=(\lambda(0) p(0), \lambda(1) p(1), \ldots, \lambda(S) p(S))]$, where $x \cdot y:=\sum_{i=1}^{\ell} x_{i} y_{i}$ denotes the dot product of two vectors $x=\left(x_{i}\right), y=\left(y_{i}\right)$ in $\mathbb{R}^{\ell}$. Besides, for every integer $n$ and every $\lambda \in \mathbb{R}^{n}, \lambda^{\perp}:=\left\{\left(x_{1}, \ldots, x_{n}\right) \in \mathbb{R}^{n}: \sum_{i=1}^{n} \lambda_{i} x_{i}=0\right\}$. Last, if $x=(x(0), \ldots, x(S))$ and $y=(y(0), \ldots, y(S))$ are two vectors of $\mathbb{R}^{1+S}$, or by extension two $(1+S)$-column vectors, then $x \leq y$ (resp. $x<<y)$ means that for every $s=0,1, \ldots, S$, one has $x(s) \leq y(s)($ resp. $x(s)<y(s))$.
} 
(iii) $\sum_{i \in I} \bar{z}_{i}=0$.

Condition $(i)$ states that $\left(\bar{x}_{i}, \bar{z}_{i}\right)$ is optimal in consumer $i$ 's budget set $B_{i}(\bar{p}, \bar{q})$. The so-called Market Clearing Conditions (ii) and (iii) guarantee the feasibility of the equilibrium allocation, and of the portfolio allocation.

We recall that even under strong assumptions on the economy, an equilibrium may fail to exist, because of possible discontinuities of the column span of $W(p, q)$, denoted $\operatorname{Im} W(p, q)$ when prices vary (see, for example, Hart [17]). A standard answer (see Duffie, Shaffer [12]) is to relax the previous equilibrium notion by the notion of pseudo-equilibrium and we refer to Aouani, Cornet [2] for another approach that assumes that the dimension of $\operatorname{Im} W(p, q)$ remains constant when prices vary. In the following, if $V$ is a Euclidean space and $k$ is an integer such that $0 \leq k \leq \operatorname{dim}(V)$, then we denote by $G^{k}(V)$ the set consisting of all the linear subspaces of $V$ of dimension $k$.

Definition 2.2. A pseudo-equilibrium of the economy $\mathcal{E}$ is a list $(\bar{x}, \bar{p}, \bar{q}, \bar{E})$ in $\mathbb{R}^{L I} \times$ $\mathbb{R}^{L} \times \mathbb{R}^{J} \times G^{J}\left(\mathbb{R}^{1+S}\right)$ such that, if we let $\bar{x}=\left(\bar{x}_{i}\right)_{i \in I}$, one has:

(i) for every $i \in I, \bar{x}_{i} \in B_{i}(\bar{p}, \bar{E})$ and $P_{i}(\bar{x}) \cap B_{i}(\bar{p}, \bar{E})=\emptyset$, where $B_{i}(\bar{p}, \bar{E}):=\left\{x_{i} \in X_{i}: \exists t_{i} \in \bar{E}, \bar{p} \square\left(x_{i}-e_{i}\right) \leq t_{i}\right\}$;

(ii) $\sum_{i \in I} \bar{x}_{i}=\sum_{i \in I} e_{i}$;

(iii) $\operatorname{Im} W(\bar{p}, \bar{q}) \subset \bar{E}$.

The following proposition shows the relationship between equilibria and pseudoequilibria.

Proposition 2.1. (a) Let $(\bar{x}, \bar{z}, \bar{p}, \bar{q})$ be an equilibrium of the economy $\mathcal{E}$ such that $\operatorname{rank} V(\bar{p})=J$, then $(\bar{x}, \bar{p}, \bar{q}, \operatorname{Im} W(\bar{p}, \bar{q}))$ is a pseudo-equilibrium of $\mathcal{E}$.

(b) Let $(\bar{x}, \bar{p}, \bar{q}, \bar{E})$ be a pseudo-equilibrium of $\mathcal{E}$ such that $\bar{E}=\operatorname{Im} W(\bar{p}, \bar{q})$, hen there exist $\bar{z}=\left(\bar{z}_{i}\right)_{i \in I} \in \mathbb{R}^{J I}$ such that $(\bar{x}, \bar{z}, \bar{p}, \bar{q})$ is an equilibrium of $\mathcal{E}$ if we additionally assume that

Non-Satiation: for every $i \in I$, for every $s_{0} \in\{0,1, \ldots, S\}$, there exists $x_{i} \in P_{i}(\bar{x})$ such that $x_{i}(s)=\bar{x}_{i}(s)$ for every $s \neq s_{0}$.

We will use the following lemma to prove Proposition 2.1.

Lemma 2.1. For every pseudo-equilibrium $(\bar{x}, \bar{p}, \bar{q}, \bar{E})$ of $\mathcal{E}$ satisfying the above NonSatiation Assumption, one has $\bar{E} \cap \mathbb{R}_{+}^{1+S}=\{0\}$, or equivalently there exists $\lambda \in$ $\mathbb{R}_{++}^{1+S}$ such that $\bar{E} \subset \lambda^{\perp}$.

Proof. First notice that the two conclusions of Lemma 2.1 are equivalent from a standard separation theorem. Let $(\bar{x}, \bar{p}, \bar{q}, \bar{E})$ be a pseudo-equilibrium and suppose that $\bar{E} \cap \mathbb{R}_{+}^{1+S} \neq\{0\}$. Then there exists $t=(t(s))_{s=0, \ldots, S}$ in $\bar{E}$ such that $t(s) \geq 0$ for every $s=0, \ldots, S$ and $t\left(s_{0}\right)>0$ for some $s_{0}, 0 \leq s_{0} \leq S$. Under the Non-Satiation Assumption for consumer 1 , there exists $x_{1} \in P_{1}(\bar{x})$ such that $x_{1}(s)=\bar{x}_{1}(s)$ for every $s \neq s_{0}$. But, from the Pseudo-equilibrium Condition $(i)$, there exists $\bar{t}_{1} \in \bar{E}$ such that $\bar{p} \square\left(\bar{x}_{1}-e_{1}\right) \leq \bar{t}_{1}$. Hence, for every integer $n$ large enough, 
$\bar{p} \square\left(x_{1}-e_{1}\right) \leq \bar{t}_{1}+n t$ and $\bar{t}_{1}+n t \in \bar{E}$. Thus $x_{1} \in P_{1}(\bar{x}) \cap B_{1}(\bar{p}, \bar{E})$ which is empty from the Pseudo-equilibrium Condition of Agent 1.

Proof of Proposition 2.1. Part (a). Let $(\bar{x}, \bar{z}, \bar{p}, \bar{q})$ be an equilibrium of the economy $\mathcal{E}$ such that $\operatorname{rank} V(\bar{p})=J$. Clearly, one has $\operatorname{rank} V(\bar{p})=\operatorname{rank} W(\bar{p}, \bar{q})=J$, which guarantees that $\operatorname{Im} W(\bar{p}, \bar{q}) \in G^{J}\left(\mathbb{R}^{1+S}\right)$ and that $(\bar{x}, \bar{p}, \bar{q}, \operatorname{Im} W(\bar{p}, \bar{q}))$ is a pseudoequilibrium of $\mathcal{E}$.

Part $(b)$. Let $(\bar{x}, \bar{p}, \bar{q}, \bar{E})$ be a pseudo-equilibrium of $\mathcal{E}$ such that $\bar{E}=\operatorname{Im} W(\bar{p}, \bar{q})$. For every $i \in I$, there exists $\bar{t}_{i} \in \bar{E}$ such that $\bar{p} \square\left(\bar{x}_{i}-e_{i}\right) \leq \bar{t}_{i}$. Summing up over $i$ these inequalities, we obtain

$$
\bar{p} \square \sum_{i \in I}\left(\bar{x}_{i}-e_{i}\right) \leq \sum_{i \in I} \bar{t}_{i}
$$

But $\sum_{i \in I}\left(\bar{x}_{i}-e_{i}\right)=0$, from the Market Clearing Condition $(i i)$, thus $\sum_{i \in I} \bar{t}_{i} \geq 0$, and we also have $\sum_{i \in I} \bar{t}_{i} \in \bar{E}$. From Lemma 2.1, one has $\bar{E} \cap \mathbb{R}_{+}^{1+S}=\{0\}$ and one deduces that $\sum_{i \in I} \bar{t}_{i}=0$. Finally, $\sum_{i \in I}\left(\bar{p} \square\left(\bar{x}_{i}-e_{i}\right)-\bar{t}_{i}\right)=0$, and each term of this sum belongs to $-\mathbb{R}_{+}^{1+S}$. Hence, each of these terms is null, that is:

$$
\bar{p} \square\left(\bar{x}_{i}-e_{i}\right)=\bar{t}_{i}, \text { for every } i \in I .
$$

Since, for every $i \in I, \bar{t}_{i} \in \bar{E}=\operatorname{Im} W(\bar{p}, \bar{q})$, there exists $z_{i} \in \mathbb{R}^{J}$ such that $\bar{t}_{i}=$ $W(\bar{p}, \bar{q}) z_{i}$. So we obtain

$$
0=\bar{p} \square \sum_{i \in I}\left(\bar{x}_{i}-e_{i}\right)=\sum_{i \in I} \bar{t}_{i}=W(\bar{p}, \bar{q})\left(\sum_{i \in I} z_{i}\right) .
$$

We now let $\bar{z}_{i}=z_{i}-(1 / I)\left(\sum_{i \in I} z_{i}\right)$ for every $i \in I$. Then, $\sum_{i \in I} \bar{z}_{i}=0$, that is, the Market Clearing Condition (iii) holds. Furthermore, we have

$$
\bar{p} \square\left(\bar{x}_{i}-e_{i}\right)=\bar{t}_{i}=W(\bar{p}, \bar{q}) z_{i}=W(\bar{p}, \bar{q}) \bar{z}_{i} .
$$

This shows that $(\bar{x}, \bar{z}, \bar{p}, \bar{q})$ is an equilibrium of $\mathcal{E}$.

\subsection{The main existence theorem}

We now set the basic assumptions on the economy $\mathcal{E}$ we will consider hereafter. We first define the set of attainable allocations of the economy, that is,

$$
\mathcal{A}(\mathcal{E}):=\left\{\left(x_{1}, \ldots, x_{I}\right) \in X_{1} \times \ldots \times X_{I}: \sum_{i \in I} x_{i}=\sum_{i \in I} e_{i}\right\}
$$

Consumption Assumption C: For every $i \in I$ :

(i) [Consumption sets] $X_{i}$ is closed, convex and bounded below, in the sense that there exists $\underline{x}_{i} \in \mathbb{R}^{L}$ such that $X_{i} \subset \underline{x}_{i}+\mathbb{R}_{+}^{L}$;

(ii) [Continuity] the correspondence $P_{i}$ is lower semicontinuous ${ }^{3}$;

\footnotetext{
${ }^{3} \mathrm{~A}$ correspondence $F$, from $X$ to $Y$, is said to be lower semicontinuous (l.s.c.) [resp. upper semicontinuous (u.s.c.)] if the set $\{x \in X: F(x) \cap U \neq \emptyset\}$ [resp. $\{x \in X: F(x) \subset U\}$ ] is open in $X$ for every open set $U \subset Y$. We define the graph of $F$ by $G(F):=\{(x, y) \in X \times Y: y \in F(x)\}$. The correspondence $F$ is said to be open-graph (resp. closed-graph) if $G(F)$ is open (resp. closed) in $X \times Y$ (for its relative topology).
} 
(iii) [Openess-type Assumption $]^{4}:$ for all $\bar{x} \in X$, for all $x_{i} \in P_{i}(\bar{x})$, for all $x_{i}^{\prime} \in$ $X_{i}, x_{i}^{\prime} \neq x_{i}$ then $\left[x_{i}^{\prime}, x_{i}\right) \cap P_{i}(\bar{x}) \neq \emptyset$;

(iv) [Convexity] for every $x \in X, P_{i}(x)$ is convex;

(v) [Irreflexivity] for every $x \in X, x_{i} \notin P_{i}(x)$;

(vi) [Non-Satiation] for every $\bar{x} \in \mathcal{A}(\mathcal{E})$, for every $s_{0} \in\{0,1, \ldots, S\}$, there exists $x_{i} \in X_{i}$ such that $x_{i}(s)=\bar{x}_{i}(s)$ for every $s \neq s_{0}$ and $x_{i} \in P_{i}(\bar{x})$;

(vii) [Strong Survival Assumption] $e_{i} \in \operatorname{int} X_{i}$.

Financial Assumption F: The mapping $p \rightarrow V(p)$ is continuous on $\mathbb{R}^{L}$.

We now state the main existence theorem which extends the existence result of Duffie-Shafer [12] to the case of agents with nonordered preferences.

Theorem 2.1. Assume the economy $\mathcal{E}$ satisfy Assumptions $\mathbf{C}$ and $\mathbf{F}$. For every $\lambda \in \mathbb{R}_{++}^{1+S}$, there exists a pseudo-equilibrium $(\bar{x}, \bar{p}, \bar{q}, \bar{E})$ of $\mathcal{E}$ such that $\bar{E} \subset \lambda^{\perp}$.

The proof of Theorem 2.1 is given in Section 3 .

When the payoff matrix $V(p)$ does not depend upon the price $p$, the financial structure is said to be nominal. We can now deduce the existence result of Cass [7], Duffie [9], Werner [30] in the case of a nominal financial structure.

Corollary 2.1. Let $\mathcal{E}=\left(\left(X_{i}, P_{i}, e_{i}\right)_{i=1 \ldots, I}, V\right)$ satisfy Assumptions $\mathbf{C}$ and $\mathbf{F}$, where $V$ is a nominal asset structure. For every $\lambda \in \mathbb{R}_{++}^{1+S}$ such that $\lambda_{0}=1$, there exists an equilibrium $(\bar{x}, \bar{z}, \bar{p}, \bar{q})$ of $\mathcal{E}$ such that $q_{j}=\sum_{s=1}^{S} \lambda_{s} V_{s}^{j}$ for every $j=1, \ldots, J$.

Proof. For every $\lambda \in \mathbb{R}_{++}^{1+S}$ such that $\lambda_{0}=1$, define $q_{j}=\sum_{s=1}^{S} \lambda_{s} V_{s}^{j}$ for every $j$. Let $J^{\prime}=\operatorname{rank} V$ and consider an economy $\mathcal{E}^{\prime}=\left(\left(X_{i}, P_{i}, e_{i}\right)_{i=1 \ldots, I}, V^{\prime}\right)$, where $V^{\prime}$ is a $S \times J^{\prime}$ full-rank submatrix of $V$. From Theorem 2.1 and Proposition 2.1 we deduce the existence of an equilibrium $\left(\bar{x}, \bar{z}^{\prime}, \bar{p}, \bar{q}^{\prime}\right)$ of $\mathcal{E}^{\prime}$. Then, one can easily modify the agents portfolios $\bar{z}^{\prime}$ and the asset prices $\bar{q}^{\prime}$ to get an equilibrium $(\bar{x}, \bar{z}, \bar{p}, \bar{q})$ of $\mathcal{E}$.

\section{Proof of Theorem 2.1}

\subsection{Proof of Theorem 2.1 under additional assumptions}

In this section, we first prove Theorem 2.1 under the following additional assumptions. The next Section 3.2 will then provide a proof of Theorem 2.1 in the general case.

Assumptions A: $(i)$ [Local Non-Satiation] For every $\bar{x}=\left(\bar{x}_{i}\right)_{i \in I} \in \mathcal{A}(\mathcal{E})$, for every $i \in I$, for every $x_{i} \in P_{i}(\bar{x})$, then $\left(\bar{x}_{i}, x_{i}\right] \subset P_{i}(\bar{x})$.

(ii) [Compactness] For every $i \in I, X_{i}$ is a compact subset of $\mathbb{R}^{L}$.

\footnotetext{
${ }^{4}$ This assumption, which is satisfied, in particular, when $P_{i}(\bar{x})$ is open in $X_{i}$ (for its relative topology) is discussed in Remark 3.1 in Section 3.2.1.
} 
3.1.1. Definition of the reaction correspondences. Let $B$ be the closed unit ball of $\mathbb{R}^{L}$, let $\mathbb{1}$ be the vector in $\mathbb{R}^{1+S}$ whose coordinates are all equal to 1 , and let $\lambda \in \mathbb{R}_{++}^{1+S}$ such that $\lambda(0)=1$. We now introduce some definitions in which $(x, p, E)$ is given in $\Pi_{i \in I} X_{i} \times B \times G^{J}\left(\lambda^{\perp}\right)$ :

- for $i=2, \ldots, I$, we consider the "augmented" budget sets:

$$
\begin{aligned}
& \beta_{i}(p, E)=\left\{x_{i} \in X_{i}: \exists t_{i} \in E, p \square\left(x_{i}-e_{i}\right) \leq t_{i}+(1-\|p\|) \mathbb{1}\right\}, \\
& \breve{\beta}_{i}(p, E)=\left\{x_{i} \in X_{i}: \exists t_{i} \in E, p \square\left(x_{i}-e_{i}\right) \ll t_{i}+(1-\|p\|) \mathbb{1}\right\} ;
\end{aligned}
$$

- for $i=1$, following the so-called "Cass trick," we consider the "augmented" Walrasian budget set (which does not depend on $E$ ):

$$
\begin{aligned}
& \beta_{1}(p, E)=\left\{x_{1} \in X_{1}:(\lambda \square p) \cdot\left(x_{1}-e_{1}\right) \leq 1-\|p\|\right\}, \\
& \breve{\beta}_{1}(p, E)=\left\{x_{1} \in X_{1}:(\lambda \square p) \cdot\left(x_{1}-e_{1}\right) \ll 1-\|p\|\right\} ;
\end{aligned}
$$

- for $i=1, \ldots, I$, we let:

$$
\Phi_{i}(x, p, E)=\left\{\begin{array}{lc}
\left\{e_{i}\right\} & \text { if } x_{i} \notin \beta_{i}(p, E) \text { and } \breve{\beta}_{i}(p, E)=\emptyset, \\
\beta_{i}(p, E) & \text { if } x_{i} \notin \beta_{i}(p, E) \text { and } \breve{\beta}_{i}(p, E) \neq \emptyset, \\
\breve{\beta}_{i}(p, E) \cap P_{i}(x) & \text { if } x_{i} \in \beta_{i}(p, E)
\end{array}\right.
$$

- for $i=0$, the revision of prices is done according to the standard rule:

$$
\Phi_{0}(x, p, E)=\left\{p^{\prime} \in B: p^{\prime} \cdot \sum_{i=1}^{I}\left(x_{i}-e_{i}\right)>p \cdot \sum_{i=1}^{I}\left(x_{i}-e_{i}\right)\right\}
$$

- for $j=1, \ldots, J$, we let:

$$
\psi_{j}(x, p, E)=\left(-\sum_{s=1}^{S} \lambda(s) V_{s}^{j}(p), V_{1}^{j}(p), \ldots, V_{S}^{j}(p)\right) .
$$

The properties of the above correspondences and mappings are summarized in the following lemma. Throughout this paper, for every Euclidean space $V$ and every integer $k \leq \operatorname{dim} V$, we will consider on the Grassmann manifold $G^{k}(V)$ the standard topology defined as follows. First, define the Stiefel manifold $V^{k}(V)$ as the collection of all $k$-tuples of linearly independent vectors of $V$. Clearly, $V^{k}(V)$ is an open subset of $V^{k}$ and it is endowed with the topology induced by the one of $V^{k}$. Then, define the mapping $f: V^{k}(V) \rightarrow G^{k}(V)$ by $f\left(e_{1}, \ldots, e_{k}\right)=\operatorname{span}\left\{e_{1}, \ldots, e_{k}\right\}$, for every $\left(e_{1}, \ldots, e_{k}\right) \in V^{k}(V)$, and define on $G^{k}(V)$ the topology as follows; a subset $U \subset G^{k}(V)$ is open if and only if its inverse image $f^{-1}(U)$ is open in $V^{k}(V)$ (see, for example, [23]).

Lemma 3.1. For every $i=0, \ldots, I$, the correspondence $\Phi_{i}$ is lower semicontinuous and has convex (possibly empty) values. For every $j=1, \ldots, J, \psi_{j}$ is a continuous mapping. 
Proof. The assertions on $\Phi_{0}$ and the $\psi_{j}, j=1, \ldots, J$, are straightforward. We notice that, for every $i=1, \ldots, I, \Phi_{i}$ has convex values and we now prove that, for every $i \in\{2, \ldots, I\}, \Phi_{i}$ is lower semicontinuous, the proof being similar for $\Phi_{1}$.

We will use the following claim, the proof of which relies on the definition of the topology of $G^{J}\left(\mathbb{R}^{1+S}\right)$ and is given in [4].

Claim 3.1. For every Euclidean space $V$ and every integer $k \leq \operatorname{dim} V$, the set $\left\{(t, E) \in V \times G^{k}(V): t \in E\right\}$ is closed.

Step $(i)$. First remark that $\breve{\beta}_{i}$ is an open-graph correspondence, so the set $A_{i}=$ $\left\{(x, p, E) \in \Pi_{k \in I} X_{k} \times B \times G^{J}\left(\lambda^{\perp}\right): \breve{\beta}_{i}(p, E) \neq \emptyset\right\}$ is open.

Step $(i i)$. We now prove that $\beta_{i}$ is a closed-graph correspondence for $i=2, \ldots, I$. Indeed, consider a sequence $\left(x_{i}^{n}, p^{n}, E^{n}\right)$ in $X_{i} \times B \times G^{J}\left(\lambda^{\perp}\right)$ such that $x_{i}^{n} \in$ $\beta_{i}\left(p^{n}, E^{n}\right)$ for every integer $n$, and suppose that $\left(x_{i}^{n}, p^{n}, E^{n}\right)$ converges to $\left(x_{i}, p, E\right) \in$ $X_{i} \times B \times G^{J}\left(\lambda^{\perp}\right)$. Then there exists a sequence $\left(t^{n}\right)$ in $\mathbb{R}^{1+S}$, such that $t^{n} \in E^{n}$ for every integer $n$, and

$$
p^{n} \square\left(x_{i}^{n}-e_{i}\right) \leq t^{n}+\left(1-\left\|p^{n}\right\|\right) \mathbb{1} .
$$

Now, remark that the sequence $\left(t^{n}\right)$ is bounded. Indeed, if it is not the case, without any loss of generality one can suppose that $\lim _{n \rightarrow \infty}\left\|t^{n}\right\|=\infty$ and that $t^{n} /\left\|t^{n}\right\|$ converges to some $t \in \mathbb{R}^{1+S}$ such that $\|t\|=1$. For $n$ large enough, we have

$$
\frac{p^{n} \square\left(x_{i}^{n}-e_{i}\right)}{\left\|t^{n}\right\|} \leq \frac{t^{n}}{\left\|t^{n}\right\|}+\frac{\left(1-\left\|p^{n}\right\|\right) \mathbb{1}}{\left\|t^{n}\right\|} .
$$

Hence, passing to limit in the above inequality, we would obtain $0 \leq t$ (since the sequences $\left(p^{n}\right)$ and $\left(x_{i}^{n}\right)$ belong to the bounded sets $B$ and $\left.X_{i}\right), t \neq 0$ (since $\|t\|=1$ ) and $t \cdot \lambda=0$ (since $t^{n} \in E^{n} \subset \lambda^{\perp}$ for every $n$ ). This contradicts $\lambda \gg 0$ and ends the proof that $\left(t^{n}\right)$ is bounded.

Now, since the sequence $\left(t^{n}\right)$ is bounded, without any loss of generality one can suppose that $\left(t^{n}\right)$ converges to some $t \in \lambda^{\perp}$. From the above inequality, we obtain

$$
p \square\left(x_{i}-e_{i}\right) \leq t+(1-\|p\|) \mathbb{1} .
$$

Besides, recalling that $t^{n} \in E^{n}$ for every $n$, from Claim 3.1, one gets $t \in E$. This proves that $\beta_{i}$ is a closed-graph correspondence for $i=2, \ldots, I$. Consequently, the set $A_{i}^{\prime}=\left\{(x, p, E) \in \Pi_{k \in I} X_{k} \times B \times G^{J}\left(\lambda^{\perp}\right): x_{i} \notin \beta_{i}(p, E)\right\}$ is open.

Step (iii). Now, notice that on the set $A_{i}, \beta_{i}$ is the closure of the open-graph correspondence $\breve{\beta}_{i}$; so $\beta_{i}$ is lower semicontinuous on $A_{i}$.

Step $(i v)$. Thus, using Steps $(i),($ ii $),($ iii $)$, the facts that $e_{i} \in \beta_{i}(p, E)$ and $\breve{\beta}_{i}(p, E) \cap$ $P_{i}(x) \subset \beta_{i}(p, E)$, one easily proves that $\Phi_{i}$ is lower semicontinuous.

3.1.2. The fixed-point argument. The existence proof relies on the following fixedpoint-like theorem by Bich and Cornet (see [4]). 
Theorem 3.1. Let $V$ be a finite dimensional Euclidean space, for $i=1, \ldots, n$, let $X_{i}$ be a nonempty, compact, convex subset of some Euclidean space, let $J$ be a positive integer such that $J \leq \operatorname{dim} V$, and let $X=\prod_{i=1}^{n} X_{i} \times G^{J}(V)$. For $i=1, \ldots, n$, let $\Phi_{i}$ be a correspondence from $X$ to $X_{i}$, which is lower semicontinuous and convex-valued (possibly empty-valued), and for $j=1, \ldots, J$, let $\psi_{j}: X \rightarrow V$ be a continuous mapping.

Then, there exists $\bar{x}=\left(\bar{x}_{1}, \ldots, \bar{x}_{n}, \bar{E}\right) \in X_{1} \times \ldots \times X_{n} \times G^{J}(V)$ such that

(a) for every $i=1, \ldots, n,\left[\right.$ either $\bar{x}_{i} \in \Phi_{i}(\bar{x})$ or $\left.\Phi_{i}(\bar{x})=\emptyset\right]$;

(b) for every $j=1, \ldots, J, \psi_{j}(\bar{x}) \in \bar{E}$.

The proof of Theorem 3.1 can be found in [4]. Remark that this theorem is a generalization first of Gale and Mas-Colell Theorem (see [14] and [15]), and also of Hirsch, Magill and Mas-Colell [18], Husseini, Lasry and Magill [19].

We will now use Theorem 3.1 to prove the existence of a pseudo-equilibrium. Let $V=\lambda^{\perp}$ and $n=I+1$. For every $i=1, \ldots, I, X_{i}$ is taken to be the consumption set of the $i$-th consumer which is convex, compact and nonempty [from Assumption C], and for $i=0, X_{0}=B$, the closed unit ball of $\mathbb{R}^{L}$. From Lemma 3.1, the correspondences $\Phi_{i}(i=0,1, \ldots, I)$ and the mappings $\psi_{j}(j=1, \ldots, J)$, defined in the previous section, satisfy the assumptions of Theorem 3.1. Consequently, from Theorem 3.1, there exists $\left(\bar{x}_{1}, \ldots, \bar{x}_{I}, \bar{p}, \bar{E}\right) \in X_{1} \times \ldots \times X_{I} \times B \times G^{J}(V)$ satisfying the above conditions $(a)$ and $(b)$ and we let $\bar{x}:=\left(\bar{x}_{1}, \ldots, \bar{x}_{I}\right)$. We first notice that, by construction and from the Irreflexivity Assumption in $\mathbf{C}, \bar{x}_{i} \notin \Phi_{i}(\bar{x}, \bar{p}, \bar{E})$ for every $i=1, \ldots, I$, and $\bar{p} \notin \Phi_{0}(\bar{x}, \bar{p}, \bar{E})$. Consequently

$\left(a^{\prime}\right)$ for every $i=0, \ldots, I, \Phi_{i}(\bar{x}, \bar{p}, \bar{E})=\emptyset$;

$\left(b^{\prime}\right)$ for every $j=1, \ldots, J, \psi_{j}(\bar{x}, \bar{p}, \bar{E}) \in \bar{E}$.

We easily deduce the following conditions (using the fact that $e_{i} \in \beta_{i}(\bar{p}, \bar{E})$ ):

$$
\begin{gathered}
\bar{x}_{i} \in \beta_{i}(\bar{p}, \bar{E}) \text { and } P_{i}(\bar{x}) \cap \breve{\beta}_{i}(\bar{p}, \bar{E})=\emptyset \text { for every } i \in I ; \\
p \cdot \sum_{i=1}^{I}\left(\bar{x}_{i}-e_{i}\right) \leq \bar{p} \cdot \sum_{i=1}^{I}\left(\bar{x}_{i}-e_{i}\right) \quad \text { for every } p \in B ; \\
\psi_{j}(\bar{x}, \bar{p}, \bar{E}) \in \bar{E} \text { for every } j=1, \ldots, J .
\end{gathered}
$$

We now let $\bar{q}=\left(\bar{q}_{1}, \ldots, \bar{q}_{J}\right)$ by $\bar{q}_{j}=\sum_{s=1}^{S} \lambda(s) V_{s}^{j}(\bar{p})$ for every $j=1, \ldots, J$.

3.1.3. The vector $(\bar{x}, \bar{p}, \bar{q}, \bar{E})$ is a pseudo-equilibrium of $\mathcal{E}$. This is a consequence of the claims proved hereafter.

Claim 3.2. $\operatorname{Im} W(\bar{p}, \bar{q}) \subset \bar{E}$.

Proof. From our choice of $\bar{q}$ we notice that for every $j=1, \ldots, J$,

$$
\psi_{j}(\bar{x}, \bar{p}, \bar{E})=\left(-\bar{q}_{j}, V_{1}^{j}(\bar{p}), \ldots, V_{S}^{j}(\bar{p})\right)=W^{j}(\bar{p}, \bar{q}),
$$


where $W^{j}(\bar{p}, \bar{q})$ denotes the $j$-th column of the matrix $W(\bar{p}, \bar{q})$. Hence, Claim 3.2 follows from the Fixed-Point Condition FP3.

The Fixed-Point Condition FP1 implies that $\bar{x}_{i} \in \beta_{i}(\bar{p}, \bar{E})$ for every $i \in I$, hence, from the definition of the sets $\beta_{i}(\bar{p}, \bar{E})$, there exists $\bar{t}_{i} \in \bar{E}$ such that:

$$
\begin{gathered}
\bar{p} \square\left(\bar{x}_{i}-e_{i}\right) \leq \bar{t}_{i}+(1-\|\bar{p}\|) \mathbb{1}, \text { for every } \mathrm{i}=2, \ldots, \mathrm{I} ; \\
(\lambda \square \bar{p}) \cdot\left(\bar{x}_{1}-e_{1}\right) \leq 1-\|\bar{p}\| .
\end{gathered}
$$

Since $\bar{t}_{i} \in \bar{E} \subset \lambda^{\perp}$ for every $i \in I$, taking the scalar product of each side of (3.1) with the vector $\lambda \in \mathbb{R}_{++}^{S+1}$, we obtain:

$$
(\lambda \square \bar{p}) \cdot\left(\bar{x}_{i}-e_{i}\right) \leq(1-\|\bar{p}\|) \sum_{s=0}^{S} \lambda(s) \text { for every } i=2, \ldots I .
$$

Claim 3.3. $\sum_{i=1}^{I} \bar{x}_{i}=\sum_{i=1}^{I} \bar{e}_{i}$.

Proof. If $\sum_{i=1}^{I}\left(\bar{x}_{i}-e_{i}\right) \neq 0$, it follows from Condition FP2 that

$$
\bar{p}=\frac{\sum_{i \in I}\left(\bar{x}_{i}-e_{i}\right)}{\left\|\sum_{i \in I}\left(\bar{x}_{i}-e_{i}\right)\right\|} .
$$

Hence, $\|\bar{p}\|=1$ and

$$
(\lambda \square \bar{p}) \cdot \sum_{i=1}^{I}\left(\bar{x}_{i}-e_{i}\right)=\sum_{s=0}^{S} \lambda(s) p(s) \cdot\left(\sum_{i}\left(\bar{x}_{i}(s)-e_{i}(s)\right)\right)=\sum_{s=0}^{S} \lambda(s) \frac{\left\|\sum_{i}\left(\bar{x}_{i}(s)-e_{i}(s)\right)\right\|^{2}}{\left\|\sum_{i}\left(\bar{x}_{i}-e_{i}\right)\right\|}
$$

and consequently

$$
(\lambda \square \bar{p}) \cdot \sum_{i=1}^{I}\left(\bar{x}_{i}-e_{i}\right) \geq\left(\min _{s} \lambda(s)\right)\left\|\sum_{i \in I}\left(\bar{x}_{i}-e_{i}\right)\right\|>0 .
$$

But summing up over $i$ the above Inequalities (3.2) and (3.3), and recalling that $\|\bar{p}\|=1$, we get

$$
(\lambda \square \bar{p}) \cdot \sum_{i=1}^{I}\left(\bar{x}_{i}-e_{i}\right) \leq(1-\|\bar{p}\|)\left(1+\sum_{s=0}^{S} \lambda(s)\right)=0,
$$

which contradicts the above inequality.

Claim 3.4. For every $s=0,1, \ldots, S, \bar{p}(s) \neq 0$.

Proof. We first notice that $\bar{p} \neq 0$. Indeed, if $\bar{p}=0$, then $\breve{\beta}_{i}(\bar{p}, \bar{E})=\beta_{i}(\bar{p}, \bar{E})=X_{i}$, for every $i \in I$. But, from Claim 3.3, $\bar{x} \in \mathcal{A}(\mathcal{E})$ and from the Non-Satiation Assumption in $\mathbf{C}$, there exists $x_{i} \in P_{i}(\bar{x})=P_{i}(\bar{x}) \cap \breve{\beta}_{i}(\bar{p}, \bar{E})$. This contradicts the Fixed-Point Condition FP1. 
We now prove that, for every $s=0, \ldots, S$, we have $\bar{p}(s) \neq 0$. Indeed, suppose that, for some $s$, one has $\bar{p}(s)=0$. From Claim 3.3, $\bar{x} \in \mathcal{A}(\mathcal{E})$, and from the Non-Satiation Assumption in $\mathbf{C}$, there exists $x_{1} \in P_{1}(\bar{x})$ such that $x_{1}\left(s^{\prime}\right)=\bar{x}_{1}\left(s^{\prime}\right)$ for $s^{\prime} \neq s$; from the Fixed-Point Condition $\mathrm{FP} 1, \bar{x}_{1} \in \beta_{1}(\bar{p}, \bar{E})$ and, recalling that $\bar{p}(s)=0$, one deduces that $x_{1} \in \beta_{1}(\bar{p}, \bar{E})$. But $\breve{\beta}_{1}(\bar{p}, \bar{E}) \neq \emptyset$, since $e_{i} \in \operatorname{int} X_{i}$ (by the Survival Assumption in $\mathbf{C}$ ), and since $\bar{p} \neq 0$ (from above). We now let $y_{1} \in \breve{\beta}_{1}(\bar{p}, \bar{E}$ ) and we notice that $\left[y_{1}, x_{1}\right) \subset \breve{\beta}_{1}(\bar{p}, \bar{E})$. Now, from the Openess-type Assumption in $\mathbf{C}$, recalling that $x_{1} \in P_{1}(\bar{x})$ and $y_{1} \in X_{1}$, we have $\left[y_{1}, x_{1}\right) \cap P_{1}(\bar{x}) \neq \emptyset$. Consequently, $P_{1}(\bar{x}) \cap \breve{\beta}_{1}(\bar{p}, \bar{E}) \neq \emptyset$, which contradicts the Fixed-Point Condition FP1.

Claim 3.5. For every $i \in I, \bar{x}_{i} \in \beta_{i}(\bar{p}, \bar{E})$ and $P_{i}(\bar{x}) \cap \beta_{i}(\bar{p}, \bar{E})=\emptyset$.

Proof. From the Fixed-Point Condition FP1, one has $\bar{x}_{i} \in \beta_{i}(\bar{p}, \bar{E})$. Now, suppose that there exists $i$ such that $P_{i}(\bar{x}) \cap \beta_{i}(\bar{p}, \bar{E}) \neq \emptyset$. Let $x_{i} \in P_{i}(\bar{x}) \cap \beta_{i}(\bar{p}, \bar{E})$. Since $e_{i} \in \operatorname{int} X_{i}$ (Survival Assumption in $\mathbf{C}$ ) and $\bar{p}(s) \neq 0$ for every $s=0, \ldots, S$, (Claim 3.4) one deduces that $\breve{\beta}_{i}(\bar{p}, \bar{E}) \neq \emptyset$ and we let $y_{i} \in \breve{\beta}_{i}(\bar{p}, \bar{E})$. We notice that $\left[y_{i}, x_{i}\right) \subset \breve{\beta}_{i}(\bar{p})$. But, $\left[y_{i}, x_{i}\right) \cap P_{i}(\bar{x}) \neq \emptyset$, from the Openess-type Assumption in $\mathbf{C}$, recalling that $x_{i} \in P_{i}(\bar{x})$ and $y_{i} \in X_{i}$. Consequently, $P_{i}(\bar{x}) \cap \breve{\beta}_{i}(\bar{p}, \bar{E}) \neq \emptyset$, which contradicts the Fixed-Point Condition FP1.

Claim 3.6. $\|\bar{p}\|=1$.

Proof. We first prove that each consumer budget constraint is binding, i.e.:

$$
\begin{gathered}
\bar{p} \square\left(\bar{x}_{i}-e_{i}\right)=\bar{t}_{i}+(1-\|\bar{p}\|) \mathbb{1} \text { for every } i=2, \ldots, I . \\
(\lambda \square \bar{p}) \cdot\left(\bar{x}_{1}-e_{1}\right)=1-\|\bar{p}\| .
\end{gathered}
$$

Indeed, suppose that (3.4) does not hold, then there exists $i=2, \ldots, I$ such that $\bar{p} \square\left(\bar{x}_{i}-e_{i}\right) \leq \bar{t}_{i}+(1-\|\bar{p}\|) \mathbb{1}$, with a strict inequality for some component, say the $s$-th component. But $\bar{x} \in \mathcal{A}(\mathcal{E})$ (Claim 3.3) and from the NonSatiation Assumption in $\mathbf{C}$ and the additional Local Non-Satiation Assumption in A, there exists $x_{i} \in X_{i}$ such that $x_{i} \in P_{i}(\bar{x}), x_{i}\left(s^{\prime}\right)=\bar{x}_{i}\left(s^{\prime}\right)$ for every $s^{\prime} \neq s$, and $\left[x_{i}, \bar{x}_{i}\right) \subset P_{i}(\bar{x})$. Besides, we can choose $x_{i}^{\prime} \in\left[x_{i}, \bar{x}_{i}\right)$ close enough to $\bar{x}_{i}$ so that $x_{i}^{\prime} \in \beta_{i}(\bar{p}, \bar{E})$. Consequently, $x_{i}^{\prime} \in P_{i}(\bar{x}) \cap \beta_{i}(\bar{p}, \bar{E})$, which contradicts the fact that $P_{i}(\bar{x}) \cap \beta_{i}(\bar{p}, \bar{E})=\emptyset$ (by Claim 3.5). This ends the proof of (3.4) and the proof of $(3.5)$ is similar.

Now, taking the scalar product of both sides of the equalities (3.4) with $\lambda \in \mathbb{R}_{++}^{S+1}$, recalling that $\bar{t}_{i} \in \bar{E} \subset \lambda^{\perp}$, we obtain

$$
(\lambda \square \bar{p}) \cdot\left(\bar{x}_{i}-e_{i}\right)=(1-\|p\|) \sum_{s=0}^{S} \lambda(s) \text { for every } i=2, \ldots, I .
$$


Summing up the equalities (3.6) (for $i=2, \ldots, I$ ) and the equality $(3.5)$ (for $i=1$ ), using the fact that $\sum_{i=1}^{I} \bar{x}_{i}=\sum_{i=1}^{I} \bar{e}_{i}$ (Claim 3.3), we get

$$
0=(\lambda \square \bar{p}) \cdot \sum_{i=1}^{I}\left(\bar{x}_{i}-e_{i}\right)=(1-\|p\|)\left(1+\sum_{s=0}^{S} \lambda(s)\right) .
$$

Consequently, $\|\bar{p}\|=1$.

Claim 3.7. For every $i \in I, \bar{x}_{i} \in B_{i}(\bar{p}, \bar{E})$ and $P_{i}(\bar{x}) \cap B_{i}(\bar{p}, \bar{E})=\emptyset$.

Proof. Since $\|\bar{p}\|=1$ (from Claim 3.6), we have $B_{i}(\bar{p}, \bar{E})=\beta_{i}(\bar{p}, \bar{E})$ for every $i=2, \ldots, I$. Hence, Claim 3.5 implies the result for every consumer $i=2, \ldots, I$.

Let us now consider the first consumer. Since $\bar{E} \subset \lambda^{\perp}$, one easily obtains that $B_{1}(\bar{p}, \bar{E}) \subset \beta_{1}(\bar{p}, \bar{E})$. From Claim 3.5, we get $\bar{x}_{1} \in \beta_{1}(\bar{p}, \bar{E})$ and $P_{1}(\bar{x}) \cap \beta_{1}(\bar{p}, \bar{E})=$ $\emptyset$, hence $P_{1}(\bar{x}) \cap B_{1}(\bar{p}, \bar{E})=\emptyset$. The proof of Claim 3.7 will thus be complete if we show that $\bar{x}_{1} \in B_{1}(\bar{p}, \bar{E})$, or the stronger assertion that

$$
\bar{p} \square\left(\bar{x}_{1}-e_{1}\right) \in \bar{E} .
$$

Since $\sum_{i=1}^{I}\left(\bar{x}_{i}-e_{i}\right)=0$ (Claim 3.3) and since the budget constraint of every consumer is binding (cf. the proof of Claim 3.6), for every $i \neq 1$, there exists $\bar{t}_{i} \in \bar{E}$ such that:

$$
\bar{p} \square\left(\bar{x}_{1}-e_{1}\right)=-\sum_{i \neq 1} \bar{p} \square\left(\bar{x}_{i}-e_{i}\right)=-\sum_{i \neq 1} \bar{t}_{i} \in \bar{E},
$$

which ends the proof of the claim.

\subsection{Proof of Theorem 2.1 in the general case}

We now give the proof of Theorem 2.1 without considering the additional assumptions A. First, given an economy $\mathcal{E}$ which satisfies Assumptions $\mathbf{C}$ and $\mathbf{F}$, we will consider a new economy $\hat{\mathcal{E}}$ with enlarged strictly preferred sets as in Gale-Mas Colell ([14], [15]). Second, we will truncate the economy $\hat{\mathcal{E}}$, to define a new economy $\hat{\mathcal{E}}^{r}$, which satisfies Assumptions C, F and A. Thus, Theorem 2.1 (with the additional Assumption A) will provide the existence of a pseudo-equilibrium of $\hat{\mathcal{E}}^{r}$, and we will check that it is also a pseudo-equilibrium of $\mathcal{E}$.

3.2.1. Enlarging the preferences. Consider an economy $\mathcal{E}=\left(\left(X_{i}, P_{i}, e_{i}\right)_{i \in I}, V\right)$. Following Gale and Mas-Colell ([14], [15]), for every $x \in \Pi_{i \in I} X_{i}$, we define the "augmented preferences" $\hat{P}_{i}$ by:

$$
\hat{P}_{i}(x):=\cup_{x_{i}^{\prime} \in P_{i}(x)}\left(x_{i}, x_{i}^{\prime}\right]=\left\{x_{i}+\lambda\left(x_{i}^{\prime}-x_{i}\right): 0<\lambda \leq 1, x_{i}^{\prime} \in P_{i}(x)\right\} \subset X_{i} .
$$

We will need the following lemma, the proof of which is similar to the one given by Gale and Mas-Colell ([14],[15])) (see also Angeloni-Cornet [1]).

Lemma 3.2. If $\mathcal{E}=\left(\left(X_{i}, P_{i}, e_{i}\right)_{i \in I}, V\right)$ satisfies Assumptions $\mathbf{C}$ and $\mathbf{F}$, then the economy $\hat{\mathcal{E}}=\left(\left(X_{i}, \hat{P}_{i}, e_{i}\right)_{i \in I}, V\right)$ satisfies Assumptions $\mathbf{C}, \mathbf{F}$ and the additional Local Non-Satiation Assumption in A. Moreover, every pseudo-equilibrium of $\hat{\mathcal{E}}$ is a pseudo-equilibrium of $\mathcal{E}$. 
Remark 3.1. The following counterexample shows that $\hat{P}_{i}$ may not have open values (relatively to $X_{i}$ ) when $P_{i}$ has open values (relatively to $X_{i}$ ). This is the main reason why we use the Openess-type Assumption $\mathbf{C}-($ iii) (instead of assuming that $P_{i}$ has open values) in both Lemma 3.2 and Theorem 2.1.

Consider the set $X=\operatorname{co}\left\{X_{0} \cup\{S, N\}\right\}$, which is the convex hull of the set $X_{0}$ and the points $N$ and $S$ defined as follows:

$$
N=(0,0,1), S=(0,0,-1), X_{0}=\left\{(x, y, z) \in \mathbb{R}^{3}: z=0,(x-1)^{2}+y^{2} \leq 1\right\},
$$

and a correspondence $P$ from $X$ to $X$ such that $P(N):=\{(x, y, z) \in X: z<0\}$. Then $P(N)$ is open for the relative topology of $X$ and $\hat{P}(N)$ is not open in $X$.

3.2.2. Truncating the economy. Consider an economy $\mathcal{E}=\left(\left(X_{i}, P_{i}, e_{i}\right)_{i \in I}, V\right)$ which satisfies Assumption C. Since the consumption sets $X_{i}$ are bounded below, we deduce that the attainable set $\mathcal{A}(\mathcal{E})$ is compact. Denoting $\hat{X}_{i}$ the projection of $\mathcal{A}(\mathcal{E})$ on $X_{i}$, we deduce that the set $\hat{X}_{i}$ is bounded for every $i \in I$. Consequently, one can choose $r>0$ large enough such that

$$
\hat{X}_{i} \subset \operatorname{int} \bar{B}(0, r) \text { for every } i \in I \text {. }
$$

For every $i \in I$, we let

$$
X_{i}^{r}=X_{i} \cap \bar{B}(0, r), \quad P_{i}^{r}(x)=P_{i}(x) \cap X_{i}^{r},
$$

and we define a new economy $\mathcal{E}^{r}$ which only differs from $\mathcal{E}$ by the fact the consumption sets $X_{i}$ have been replaced by the above sets $X_{i}^{r}$ and the correspondences $P_{i}$ by $P_{i}^{r}$. To summarize, we let the truncated economy of $\mathcal{E}$ be

$$
\mathcal{E}^{r}:=\left(\left(X_{i}^{r}, P_{i}^{r}, e_{i}\right)_{i \in I}, V\right) .
$$

Then, one can easily prove the following lemma:

Lemma 3.3. Let $\mathcal{E}$ satisfy Assumptions $\mathbf{C}, \mathbf{F}$, and the Local Non-Satiation Assumption in $\mathbf{A}$ and let $r>0$ be chosen as above. Then $\mathcal{E}^{r}$ satisfies Assumptions $\mathbf{C}, \mathbf{F}$ and $\mathbf{A}$ and every pseudo-equilibrium of $\mathcal{E}^{r}$ is a pseudo-equilibrium of $\mathcal{E}$.

3.2.3. Proof of Theorem 2.1 in the general case. Let $\mathcal{E}=\left(\left(X_{i}, P_{i}, e_{i}\right)_{i \in I}, V\right)$ satisfy Assumptions $\mathbf{C}$ and F. Applying Lemma 3.2 and Lemma 3.3, one can define the enlarged economy $\hat{\mathcal{E}}$ which satisfies Assumptions $\mathbf{C}, \mathbf{F}$ and the local Non Satiation Assumption in $\mathbf{A}$ and one can choose $r>0$ large enough so that the truncated enlarged economy $\hat{\mathcal{E}}^{r}$ satisfies Assumptions C, F and A. From Section 3.1 (providing the proof of Theorem 2.1 with the additional Assumptions A), there exists a pseudo-equilibrium $(\bar{x}, \bar{p}, \bar{q}, \bar{E})$ of $\hat{\mathcal{E}}^{r}$ such that $\bar{E} \subset \lambda^{\perp}$. From Lemma 3.2 and Lemma $3.3,(\bar{x}, \bar{p}, \bar{q}, \bar{E})$ is also a pseudo-equilibrium of the economy $\mathcal{E}$. This ends the proof of Theorem 2.1. 


\section{References}

[1] L. Angeloni, B. Cornet, Existence of financial equilibria in a multi-period stochastic economy. Advances in Mathematical Economics 8 (2006), 1-31.

[2] Z. Aouani, B. Cornet, Existence of financial equilibria with restricted participation. Working Paper, University of Kansas (2007).

[3] K. J. Arrow, G. Debreu, Existence of an equilibrium for a competitive economy. Econometrica 22 (1954), 265-290.

[4] P. Bich, B. Cornet, Fixed-point-like theorems on subspaces. Fixed Point Theory and Applications 3 (2004), 159-171.

[5] Y. Balasko, Foundations of the Theory of General Equilibrium. Academic Press, Boston (1988).

[6] L. E. J. Brouwer, Über abbildung von mannigfaltigkeiten. Mathematische Annalen 71 (1912), 97-115.

[7] D. Cass, Competitive equilibrium with incomplete financial markets. Journal of Mathematical Economics 42 (2006), 384-405, first published as CARESS Working paper 84-09, University of Pennsylvania.

[8] B. Cornet, R. Gopalan, Restricted participation and arbitrage in a multiperiod model. Economic Theory, forthcoming.

[9] D. Duffie, Stochastic equilibria with incomplete financial markets. Journal of Economic Theory 41 (1987), 404-416.

[10] E. Dierker, Topological Methods in Walrasian Economics. Lecture Notes in Economics and Mathematical Systems, Springer, Berlin 92 (1974).

[11] G. Debreu, Theory of Value. Wiley, New-York (1959).

[12] D. Duffie, W. Shaffer, Equilibrium in incomplete markets I: basic model of generic existence. Journal of Mathematical Economics 14 (1985), 285-300.

[13] S. Eilenberg, D. Montgomery, Fixed point theorems for multivalued transformations. American Journal of Mathematics 68 (1946), 214-222.

[14] D. Gale, A. Mas-Colell, An equilibrium existence theorem for a general model without ordered preferences. Journal of Mathematical Economics 2 (1975), 9-15.

[15] D. Gale, A. Mas-Colell, Corrections to an equilibrium existence theorem for a general model without ordered preferences. Journal of Mathematical Economics 6 (1979), 297298.

[16] J. Geanakoplos, An introduction to general equilibrium with incomplete asset markets. Journal of Mathematical Economics 19 (1990), 1-38.

[17] O. Hart, On the optimality of equilibrium when the market structure is incomplete. Journal of Economic Theory 11 (1975), 418-443.

[18] M. Hirsch, M. Magill, M. Mas-Colell, A geometric approach to a class of equilibrium existence theorems. Journal of Mathematical Economics 19 (1987), 95-106.

[19] S. Y. Husseini, J. M. Lasry, M., Magill, Existence of equilibrium with incomplete markets. Journal of Mathematical Economics 19 (1986), 39-67.

[20] S. Kakutani, A generalisation of Brouwer's fixed point theorem. Duke Mathematical Journal 8 (1941), 457-9. 
[21] M. Magill, M. Quinzii, Theory of Incomplete Markets. MIT Press, Cambridge, MA (1996).

[22] A. Mas Colell, The Theory of General Economic Equilibrium : a Differentiable Approach. Cambridge University Press (1985).

[23] J. W. Milnor, J. D. Stasheff, Characteristic classes. Princeton Univ. Press, Ann. of Math. Studies 76 (1974).

[24] R. Radner, Existence of equilibrium plans, prices, and price expectations. Econometrica 40 (1972), 289-303.

[25] S. Smale, Global analysis and economics I, Pareto optimum and a generalization of Morse Theory. in Dynamical Systems, M. Peixoto, ed., Academic Press, New York (1973).

[26] S. Smale, Global analysis and economics, IIA, extension of a theorem of Debreu. Journal of Mathematical Economics 1 (1974), 1-14.

[27] S. Smale, Global analysis and economics, III, Pareto optima and price equilibrium. Journal of Mathematical Economics 1 (1974), 107-117.

[28] S. Smale, Global analysis and economics, IV, finiteness and stability of equilibria with general consumption sets and production. Journal of Mathematical Economics 1 (1974), 119-127.

[29] S. Smale, Global analysis and economics, V, Pareto theory with constraints. Journal of Mathematical Economics 1 (1974), 213-221.

[30] J. Werner, Equilibrium in economies with incomplete financial markets. Journal of Economic Theory 36 (1985), 110-119.

Philippe Bich

Paris School of Economics, Centre d'Economie de la Sorbonne

Université Paris 1 Panthéon Sorbonne

75647 Paris Cedex 13, France

e-mail: bich@univ-paris1.fr

Bernard Cornet

Paris School of Economics, Centre d'Economie de la Sorbonne

Université Paris 1 Panthéon Sorbonne

75647 Paris Cedex 13, France

and

Department of Economics

University of Kansas

66045 Kansas, USA

e-mail: cornet@univ-paris1.fr, cornet@ku.edu 